\title{
HST/STIS low dispersion spectroscopy of three Compact Steep Spectrum sources
}

\section{Evidence for jet-cloud interaction}

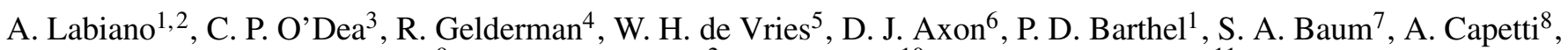 \\ R. Fanti ${ }^{9}$, A. M. Koekemoer ${ }^{2}$, R. Morganti ${ }^{10}$, and C. N. Tadhunter ${ }^{11}$
}

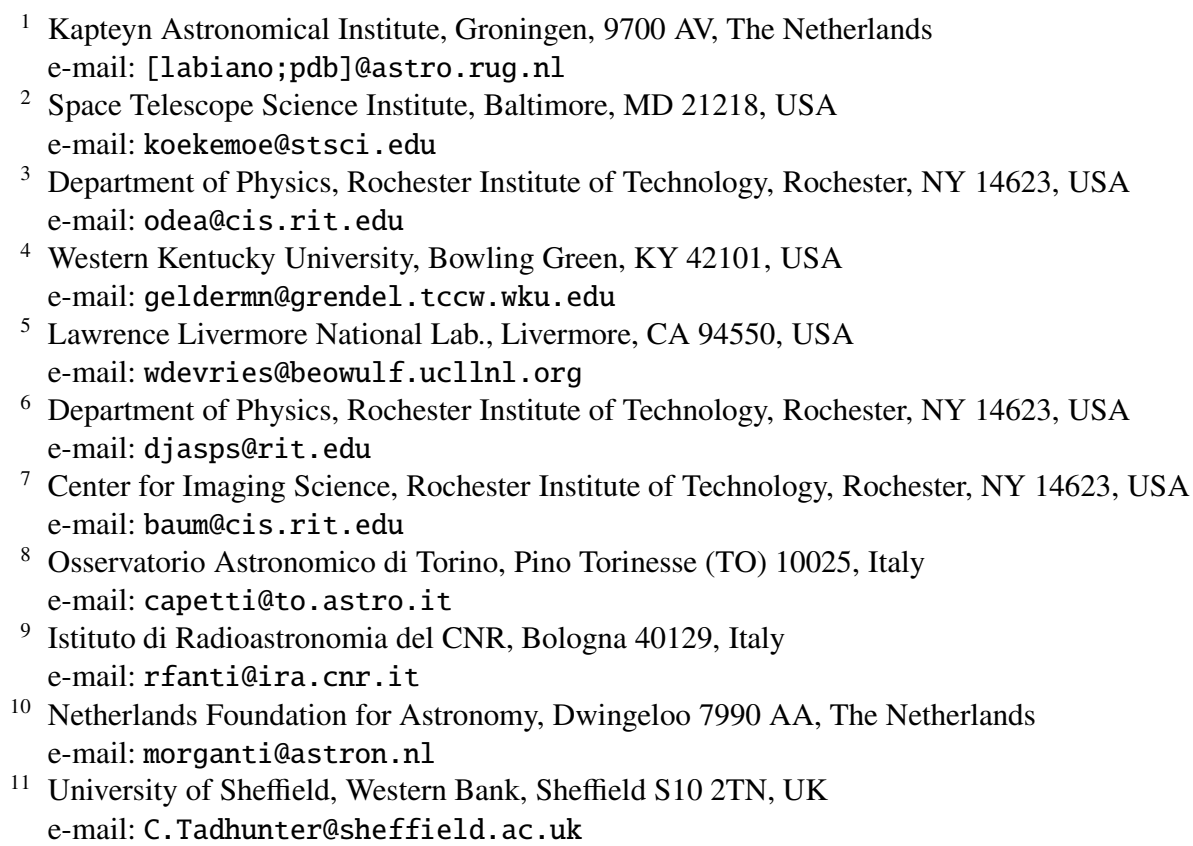

Received 23 November 2004 / Accepted 4 March 2005

\begin{abstract}
We present Hubble Space Telescope Imaging Spectrograph long-slit spectroscopy of the emission line nebulae in the compact steep spectrum radio sources 3C 67, 3C 277.1, and 3C 303.1. We derive BPT (Baldwin- Philips-Terlevich; Baldwin et al. 1981) diagnostic emission line ratios for the nebulae which are consistent with a mix of shock excitation and photoionization in the extended gas. In addition, line ratios indicative of lower ionization gas are found to be associated with higher gas velocities. The results are consistent with a picture in which these galaxy scale radio sources interact with dense clouds in the interstellar medium of the host galaxies, shocking the clouds thereby ionizing and accelerating them.
\end{abstract}

Key words. galaxies: active - galaxies: individual: 3C 67 - galaxies: individual: 3C 277.1 - galaxies: individual: 3C 303.1 galaxies: quasars: emission lines

\section{Introduction}

Powerful radio galaxies play a critical role in our understanding of both galaxy evolution and the phenomenon of activity in galactic nuclei. Yet we know little about how the radio galaxies are born and how they subsequently evolve. Recent work has identified the GHz Peaked Spectrum (GPS) and Compact Steep Spectrum (CSS) radio sources as the most likely candidates for the progenitors of the large scale powerful classical double (FR2) sources (e.g. O'Dea et al. 1991; Fanti et al. 1990, 1995; Readhead et al. 1996b,a; O'Dea \& Baum 1997); for a review see O'Dea (1998). The GPS and CSS sources are powerful but compact radio sources whose spectra are generally simple and convex with peaks near $1 \mathrm{GHz}$ and $100 \mathrm{MHz}$ respectively. The GPS sources are contained within the extent of the optical narrow emission line region ( $\lesssim 1 \mathrm{kpc}$ ) while the CSS sources are contained within the host galaxy ( $\$ 15 \mathrm{kpc})$. 
Current models for the evolution of powerful radio galaxies suggest that these sources propagate from the $\sim 10 \mathrm{pc}$ to Mpc scales at roughly constant velocity through an ambient medium which declines in density as $\rho(R) \propto R^{-2}$ while the sources decline in radio luminosity as $L_{\mathrm{rad}} \propto R^{-0.5}$ (Fanti et al. 1995; Begelman 1996; Readhead et al. 1996a; De Young 1997; Kaiser et al. 1997; Kaiser \& Alexander 1997; Snellen et al. 2000). Such a scenario is consistent with the observed number densities of powerful radio sources as a function of linear size (from tens of parsecs to hundreds of kpc) (e.g. O'Dea \& Baum 1997; Fanti et al. 2001). However, the situation must be more complicated than this simple picture. We give two reasons: (1) the GPS and CSS sources must interact with the host galaxy as they propagate through it. The discovery of emission line gas aligned with and presumably co-spatial with the CSS radio sources and the presence of broad and complex integrated emission line profiles (Gelderman \& Whittle 1994) indicates that the radio source is strongly interacting with the ambient gas (de Vries et al. 1997, 1999; Axon et al. 2000). Therefore, we would expect shocks to contribute strongly to the ionization of the gas (e.g. Bicknell et al. 1997); (2) the GPS sources are observed to have expansion velocities several times higher (Conway 1998, private communication) than the estimated advance speeds of large scale classical doubles (Alexander \& Leahy 1987). This would require the evolving GPS sources to decelerate as they propagate though the host galaxy and would require the radio sources to dim faster than the simple models predict. It may be that the deceleration takes place via interaction with ambient gas (see De Young 1993; Carvalho 1994, 1998).

We are carrying out a study of the kinematics and ionization of the aligned emission line nebulae in three CSS radio sources: 3C 67 (galaxy, $z=0.310$, linear size $D=10.1 \mathrm{Kpc}$ ), 3C 277.1 (quasar, $z=0.321, D=6.9 \mathrm{Kpc}$ ), 3C 303.1 (galaxy, $z=0.267, D=6.2 \mathrm{Kpc})^{1}$ with HST/STIS long-slit spectroscopy. In O'Dea et al. (2002) we presented our results on the kinematics of the [O III] $\lambda 5007$ emission line and found complex emission line profiles and large differences in velocity offset on the two sides of the nucleus, suggesting that the cloud motions are being driven by shocks induced by the expanding radio lobes. In O'Dea et al. (2003) we discussed two models for the cloud kinematics - (1) acceleration by the radio source bow shock and (2) acceleration by the post bow shock wind, and concluded that the bow shock acceleration was favored. Here we present the results of our low dispersion spectra of several diagnostic emission lines. We compare our results with shock and photo-ionization models and examine the relationships between the ionization diagnostics and the gas kinematics.

\section{Observations}

We obtained STIS long slit spectra through the $52^{\prime \prime} \times 0.1^{\prime \prime}$. slit through several gratings. The instrumental parameters are summarized in Table 1 . The observations through the medium dispersion G750M grism has been used primarily to study the

\footnotetext{
${ }^{1}$ We adopt a Hubble constant of $H_{0}=75 \mathrm{~km} \mathrm{~s}^{-1} \mathrm{Mpc}^{-1}$ and a deceleration parameter of $q_{0}=0.0$.
}

Table 1. Journal of HST/STIS observations.

\begin{tabular}{llccl}
\hline \hline Source & Grism & $\begin{array}{c}\text { Central } \lambda \\
\AA\end{array}$ & $\begin{array}{c}\text { PA } \\
\text { degrees }\end{array}$ & Time \\
\hline 3C 67 & G750L & 7751 & 17 & 2160 \\
3C 67 & G430L & 4300 & 17 & 2660 \\
3C 277.1 & G750L & 7751 & 311 & 2340 \\
3C 277.1 & G430L & 4300 & 311 & 2800 \\
3C 303.1 & G750L & 7781 & 331 & 2900 \\
3C 303.1 & G430L & 4300 & 331 & 5300 \\
\hline
\end{tabular}

HST proposal ID $=8104$. The slit was placed parallel to the radio source axis.

kinematics of the nebula and are discussed by O'Dea et al. (2002). For the study of the ionization, we observed through the low dispersion G750L (5236 to $10266 \AA$ ) and G430L (2900 to $5700 \AA$ ) grisms centered on $7751 \AA$ and $4300 \AA$, respectively, with a spectral resolution of $5 \AA /$ pixel and a Line Spread Function (LSF) of 2.0 pixels resulting in a velocity resolution of $\sim 700 \mathrm{~km} \mathrm{~s}^{-1}$ at the center of the blue side of the spectra and $\sim 390 \mathrm{~km} \mathrm{~s}^{-1}$ at the center of the red side ${ }^{2}$. The spatial resolution is given by the Point Spread Function (PSF) of the detector, with a $F W H M$ of 2.3 pixels.

We integrated for one orbit on each slit position ( 2500-3000 s) with the orientation taken roughly parallel to the radio source axis. In each source one slit was centered on the source nucleus and aligned along the radio axis. In 3C 67 and $3 \mathrm{C} 303.1$, where the emission line gas is slightly misaligned with respect to the radio axis, we rotated the slit to place it along the emission-line nebula axis, rather than the radio source axis.

\section{Data reduction}

The standard STIS reduction pipeline was used to remove detector signatures such as bias, dark current, and flat fielding and to apply the flux calibration. Cosmic ray hits can be quite numerous over the course of orbit-long exposures. We therefore split each exposure per orbit into two equal-length parts in order to allow removal of cosmic rays. Any surviving cosmic rays were removed and replaced by the average of the flux values in the adjacent pixels around the cosmic ray.

To correct the spatial dispersion of the light produced by the spectrograph (given by the PSF) we have averaged (weighted by the flux errors given by the spectrograph) three pixels in the spatial direction for every source. We also averaged all the rows in each lobe for each source, to produce an average lobe spectrum for each side of the source.

We used the IRAF/STSDAS Specfit (Kriss 1994) software to fit Gaussians to the profile of each emission line in our sources (Table 2 and Figs. 1-3) measuring each line's integrated flux, full width at half maximum $(F W H M)$ and central wavelength (i.e., velocity offset relative to the nucleus). Some constraints were adopted to reduce the number of free parameters and limit our options to "physically consistent models", as follows: we classified the detected lines in the spectrum as either high or low ionization (see Table 2). We required all

\footnotetext{
${ }^{2}$ Corrected for redshift.
} 
Table 2. Emission lines master list.

\begin{tabular}{|c|c|c|c|c|c|c|c|c|c|c|c|}
\hline \multirow[b]{2}{*}{ Line } & \multirow[b]{2}{*}{$\lambda(\AA)$} & \multirow[b]{2}{*}{ Ion. } & \multicolumn{3}{|c|}{$3 \mathrm{C} 67$} & \multicolumn{3}{|c|}{ 3C 277.1} & \multicolumn{3}{|c|}{ 3C 303.1} \\
\hline & & & $\mathrm{Nuc}$ & $\mathrm{S}$ & $\mathrm{N}$ & Nuc & $S$ & $\mathrm{~N}$ & Nuc & $\mathrm{S}$ & $\mathrm{N}$ \\
\hline [Mg II] & 2799.12 & Low & $24.0 \pm 0.7$ & - & - & $2738 \pm 40$ & - & - & - & - & - \\
\hline$[\mathrm{Ne} \mathrm{V}]$ & 3425.90 & High & $143.9 \pm 6.3$ & $23.2 \pm 1.4$ & - & $427 \pm 18$ & - & - & - & - & - \\
\hline [O II] & 3727.37 & Low & $111.2 \pm 4.6$ & $57.1 \pm 9.8$ & $20.3 \pm 1.5$ & $188.9 \pm 15$ & $65.0 \pm 1.8$ & $33.8 \pm 0.6$ & $275.4 \pm 3.6$ & $144.2 \pm 1.4$ & $63.1 \pm 1.3$ \\
\hline [Ne III] & 3868.76 & High & $133.6 \pm 6.0$ & - & - & $627 \pm 17$ & - & - & $52.4 \pm 1.8$ & - & - \\
\hline [Ne III] & 3967.47 & High & $13.2 \pm 4.1$ & - & - & $412 \pm 26$ & - & - & - & - & - \\
\hline $\mathrm{H} \delta$ & 4101.73 & Low & - & - & - & $212.8 \pm 3.3$ & - & - & - & - & - \\
\hline $\mathrm{H} \gamma$ & 4340.47 & Low & - & - & - & $343.1 \pm 4.0$ & - & - & $25.1 \pm 1.1$ & - & - \\
\hline [O III] & 4363.21 & High & & $15.4 \pm 1.1$ & - & $332.7 \pm 3.4$ & - & - & - & - & - \\
\hline [He II] & 4685.68 & Low & - & - & - & $57.7 \pm 3.7$ & - & - & $23.7 \pm 1.1$ & - & - \\
\hline $\mathrm{H} \beta$ & 4861.33 & Low & $95.9 \pm 2.7$ & $33.4 \pm 1.4$ & - & $281.0 \pm 4.1$ & $30.4 \pm 1.6$ & - & $72.7 \pm 1.5$ & $13.7 \pm 0.6$ & $18.9 \pm 0.8$ \\
\hline [O III] & 4958.92 & High & $54.0 \pm 3.2$ & $67.9 \pm 1.2$ & $25.2 \pm 0.9$ & $1031.3 \pm 4.7$ & $27.4 \pm 1.2$ & $35.7 \pm 0.8$ & $258.9 \pm 1.6$ & $32.3 \pm 0.6$ & $35.9 \pm 0.6$ \\
\hline [O III] & 5006.85 & High & $1566.8 \pm 5.7$ & $196.7 \pm 1.4$ & $73.0 \pm 1.0$ & $2998.3 \pm 9.2$ & $79.7 \pm 1.3$ & $103.9 \pm 0.9$ & $752.1 \pm 2.8$ & $95.3 \pm 0.6$ & $104.4 \pm 0.7$ \\
\hline$[\mathrm{He} \mathrm{I}]$ & 5875.70 & Low & $45.2 \pm 3.1$ & - & - & $98.4 \pm 4.5$ & - & - & - & - & - \\
\hline [O I $]$ & 6300.31 & Low & - & - & - & - & - & - & - & - & - \\
\hline [N II] & 6548.06 & Low & - & - & - & $181.6 \pm 5.1$ & - & - & $59.6 \pm 2.7$ & $16.8 \pm 0.8$ & - \\
\hline $\mathrm{H} \alpha$ & 6562.82 & Low & $372.0 \pm 3.7$ & $103.4 \pm 1.7$ & $37.6 \pm 1.3$ & $2923.3 \pm 12.6$ & $94.1 \pm 2.5$ & $88.7 \pm 2.0$ & $210.6 \pm 2.3$ & $39.7 \pm 0.9$ & $59.4 \pm 1.1$ \\
\hline$[\mathrm{N}$ II] & 6583.39 & Low & $75.2 \pm 3.3$ & $58.9 \pm 1.6$ & - & $527.4 \pm 5.9$ & $4.9 \pm 2.4$ & $36.1 \pm 2.0$ & $193.5 \pm 3.1$ & $48.9 \pm 0.9$ & $34.5 \pm 1.0$ \\
\hline [S II] & 6716.42 & Low & - & - & - & $160.6 \pm 5.2$ & - & - & $56.2 \pm 16$ & $32.7 \pm 1.1$ & - \\
\hline [S II] & 6731.78 & Low & - & - & - & - & - & - & - & - & - \\
\hline
\end{tabular}

List of the emission lines we searched for in our sample. The first column gives the species; the second column the rest-frame wavelength; the third column the ionization status. For wavelength and ionization references see Eracleous \& Halpern (2004). For each source we present the integrated flux (in $10^{-16} \mathrm{erg} \mathrm{s}^{-1} \mathrm{~cm}^{-2}$ arcsec ${ }^{-2}$ ) for the detected lines in the nucleus (Nuc) and southern (S) or northern (N) lobes. Nondetections are indicated by "-". For the lobes we have used the spatially averaged spectra. However, a line marked in the table as a non detection in the averaged spectra might have been detected in a couple of very localized positions. We require $3 \sigma$ for a detection. See also Figs. 1-3 for spectra of the sources.

gas with same ionization state to have the same FWHM and velocity offset, and took as as free parameters the widths and velocities of $\mathrm{H} \beta$ and [O III] $] \lambda 5007$. The integrated fluxes of every line were free to vary except for those with known ratios (from atomic physics, see e.g., Osterbrock 1989), in this case: the [N II] $\lambda \lambda 6548,84$ and [O III] $\lambda \lambda 4959,5007$ doublets. We used a power law to fit the continuum. Whenever this model was not accurate enough in parts of the spectrum, we limited the fit to the adjacent regions of each emission line, improving our continuum model and producing accurate fits for the line profiles. Only a few of the potential emission lines (Table 2) were detected in the extended nebulae (signal to noise ratio $>3$ ) - the [O II] $\lambda \lambda$ 3727, 29 doublet, $\mathrm{H} \alpha, \mathrm{H} \beta$, [O III] $\lambda \lambda$ 4959, 5007 and the [N II] $\lambda \lambda 6548,84,\left[\mathrm{~S}\right.$ II] $\lambda \lambda 6716,31$ doublets $^{3}$. Upper limits were defined to be three times the noise of the spectrum, multiplied by the width of the line: $3 \times \mathrm{rms} \times F W H M$. We used the width we measured in the resolved lines of the same ionization status.

Specfit is known to overestimate the errors for flux and central wavelength measurements in our data. To improve the estimation of our error bars we created over one hundred artificial spectra consisting of a perfect Gaussian with known random white Gaussian noise added, which covered the parameter

\footnotetext{
${ }^{3}$ We have detected the $[\mathrm{S} \mathrm{II}] \lambda \lambda 6716,31$ emission in very few points of the sources and our low wavelength resolution does not allow us to deblend the doublet components.
}

space of our data in signal to noise ratios and resolution. We created ten artificial spectra for each different signal to noise ratio and ran Specfit on each spectrum. We compared the Specfit derived errors with those expected based on the parameters of the artificial spectra and estimated a correction to the Specfit errors. The final step in the data reduction was correcting the data for reddening, using the Galactic dereddening curve in Cardelli et al. (1989) and the measured Galactic extinction values of Schlegel et al. (1998).

O'Dea et al. (2002) searched for the possible presence of faint, broad wings ( $\gtrsim 1500-2000 \mathrm{~km} \mathrm{~s}^{-1}$ ) using the low dispersion data, that might have been undetected in the higher dispersion data. They found no strong evidence for broad, nonGaussian components substantially above $\sim 1500 \mathrm{~km} \mathrm{~s}^{-1}$ in any of these galaxies.

\section{Results}

We have detected several lines in the extended nebulae of all three CSS sources - [O II], [O III], H $\beta, \mathrm{H} \alpha$, and [N II]. We have used these lines to determine the standard diagnostics of ionization and compared them to the predictions of shock and photoionization models. We have also compared the ionization levels with the gas kinematics, as presented and discussed by O'Dea et al. (2002). 

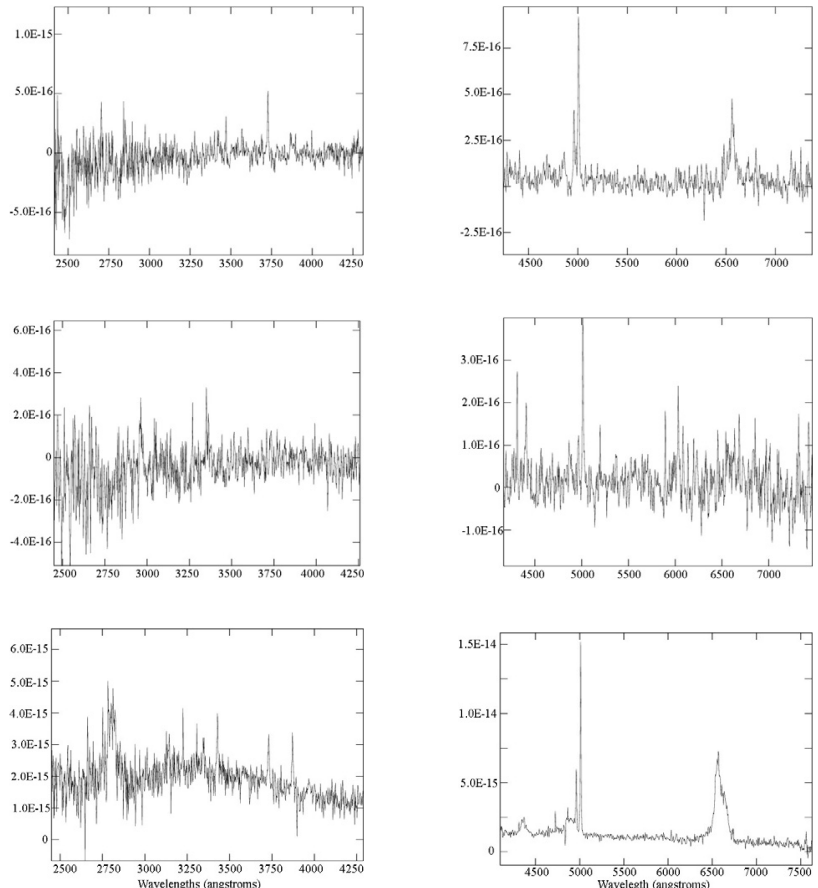

Fig. 1. Spectra of the lobes and nucleus of 3C 67. Left is G430L and right is G750L. The top row corresponds to the spatially averaged spectra of the southern lobe, the middle row to the averaged spectra of the northern lobe and the bottom row corresponds to the nucleus. We have trimmed the sides of some spectra for clarity but all the wavelengths of interest are shown. The units of flux are $\operatorname{erg~s}^{-1} \mathrm{~cm}^{-2} \operatorname{arcsec}^{-2} \AA^{-2}$.
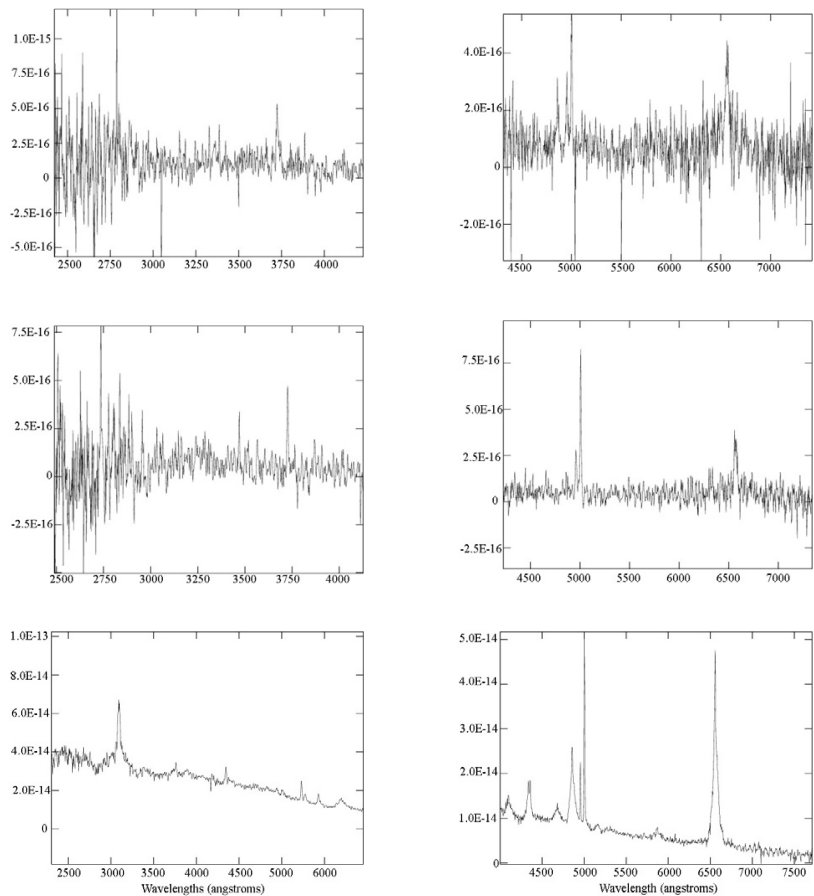

Fig. 2. Spectra of the lobes and nucleus of 3C 277.1. Left is G430L and right is G750L. The top row corresponds to the spatially averaged spectra of the southern lobe, the middle row to the averaged spectra of the northern lobe and the bottom row corresponds to the nucleus. We have trimmed the sides of some spectra for clarity but all the wavelengths of interest are shown. The units of flux are $\operatorname{erg~s}^{-1} \mathrm{~cm}^{-2} \operatorname{arcsec}^{-2} \AA^{-2}$.
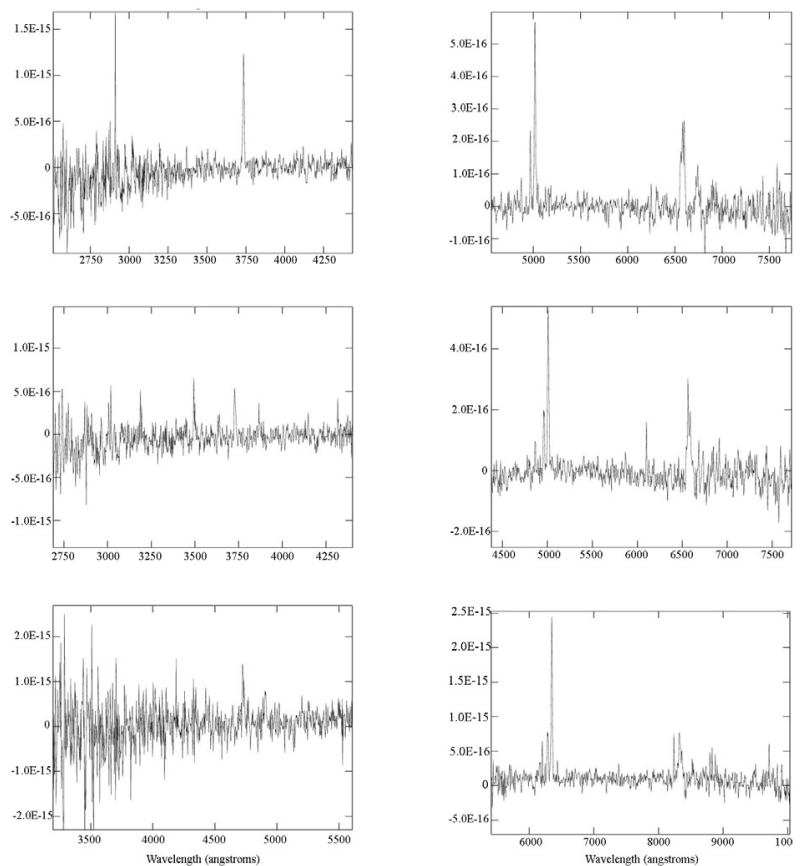

Fig. 3. Spectra of the lobes and nucleus of 3C 303.1. Left is G430L and right is $\mathrm{G} 750 \mathrm{~L}$. The top row corresponds to the spatially averaged spectra of the southern lobe, the middle row to the averaged spectra of the northern lobe and the bottom row corresponds to the nucleus. We have trimmed the sides of some spectra for clarity but all the wavelengths of interest are shown. The units of flux are $\operatorname{erg~s}^{-1} \mathrm{~cm}^{-2} \operatorname{arcsec}^{-2} \AA^{-2}$.

\subsection{Ionization diagnostics}

Given our line detections we were able to construct one of the key diagnostic Baldwin-Philips-Terlevich (BPT) diagrams (Baldwin et al. 1981; Veilleux \& Osterbrock 1987; Moy \& Rocca-Volmerange 2002), namely the $[\mathrm{OII}] /[\mathrm{O} I I I]$ vs. $[\mathrm{O} \mathrm{III}] / \mathrm{H} \beta$ diagram (Figs. 4-6). Other potentially useful BPT diagrams (e.g., [S II]/H $\alpha$ vs. [N II] 6583/H $\alpha$, $[\mathrm{O} \mathrm{II}] \lambda \lambda \quad 3727+29 /[\mathrm{O}$ III $] 5007$ vs. [N II] $6583 / \mathrm{H} \alpha$, [O III $] 5007 / \mathrm{H} \beta$ vs. $[\mathrm{S} \mathrm{II}] / \mathrm{H} \alpha$, etc.) were not possible given the limited number of detected lines. We have compared our results with the two main classes of ionization mechanisms: shock ionization using MAPPINGS III (Kewley et al. 2003, in preparation; Dopita \& Sutherland 1996) and AGN photo-ionization using CLOUDY (Ferland et al. 1998). The MAPPINGS shock code calculates the spectra from gas which has been directly ionized by the shock (pure shock models) and unshocked gas which has been ionized by the radiation emitted by the hot shocked gas (pure precursor models). In our BPT diagrams (Figs. 4-6) we show the predicted line ratios calculated by MAPPINGS for both a pure precursor and pure shock model, as well as linear combinations of these two (30\%, 50\%, 70\% contribution to the emission line luminosity from shocks), including a range of magnetic fields (MAPPINGS's magnetic parameter from 0.5 to 10) and shock velocities from 100 to $1000 \mathrm{~km} \mathrm{~s}^{-1}$. In addition we include the predicted line ratios calculated by CLOUDY for a range of ionization parameter ( $\log U$ ranging from -2 to -3.8 ) and cloud density $(\log n=0,2,4)$. We note that "real" radio 


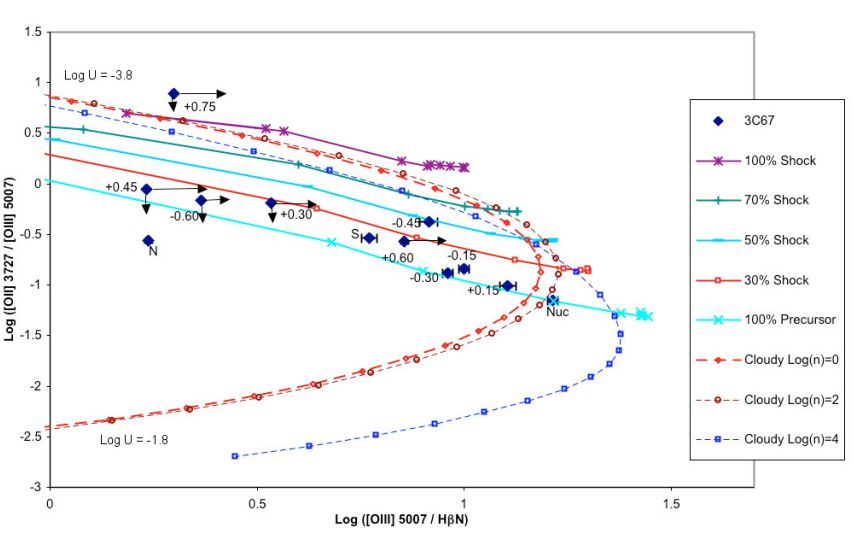

Fig. 4. 3C 67. Reddening-corrected [O II] $\lambda \lambda 3727+29 /[\mathrm{O}$ III] $\lambda 5007$ vs. [O III] $\lambda 5007 / \mathrm{H} \beta$ intensity ratios. Symbols for the models are shown in key to the figure. Data is shown for the nucleus (Nuc) and at each 0.15 arcsec along the slit in the extended region, labeled with their distance to the nucleus. We also show the points for the averaged extended emission in the northern $(\mathrm{N})$ and southern (S) lobes. The arrows in the plot represent those points where lines were not detected, using an upper limit of $(3 \times \mathrm{rms} \times F W H M)$. We compare our results with the two main classes of ionization mechanisms: MAPPINGS III (Kewley et al. 2003, in preparation; Dopita \& Sutherland 1996) shock models (solid lines) and CLOUDY (Ferland et al. 1998) AGN photo-ionization models (dashed lines). For the shock models from MAPPINGS we included both pure precursor and pure shock models, as well as linear combinations of these two $(30 \%, 50 \%, 70 \%$ contribution to the emission line luminosity from shocks). The results do not depend on magnetic field strength, and so for simplicity we show models with $B=10$. Shock velocity in this models ranges from 100 to $1000 \mathrm{~km} \mathrm{~s}^{-1}$ and increases to the right. For the CLOUDY AGN photoionization models we include a range of ionization parameter ( $\log U$ ranging from -1.8 to -3.8 ) and cloud density $(\log n=0,2,4)$.

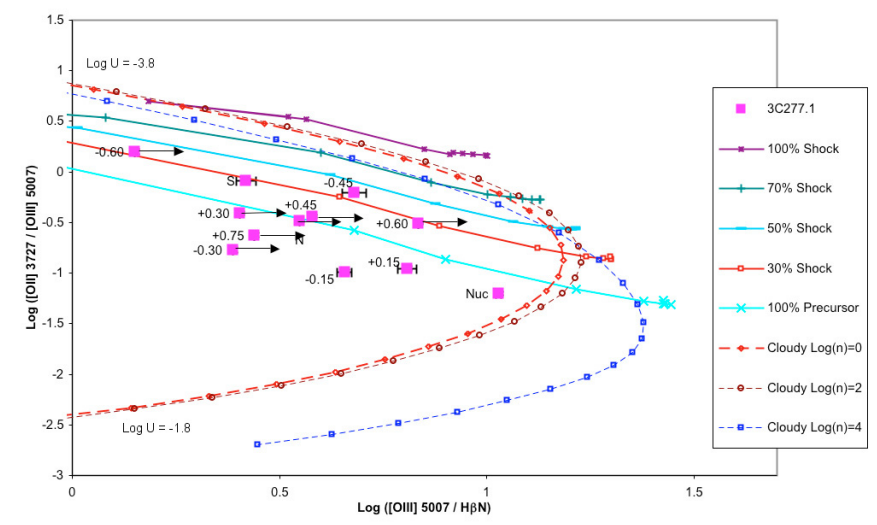

Fig. 5. Same as Fig. 4, but for 3C 277.1.

galaxies are probably more complicated than assumed in the MAPPINGS and CLOUDY calculations and suggest that the results be taken with caution.

We find that the data points tend to spread across the diagrams (Figs. 4-6) covering about one dex in both dimensions. The nuclei lie to the lower right of the distribution of points (with the highest values of $[\mathrm{O} \mathrm{III]}] / \mathrm{H} \beta$, and lowest

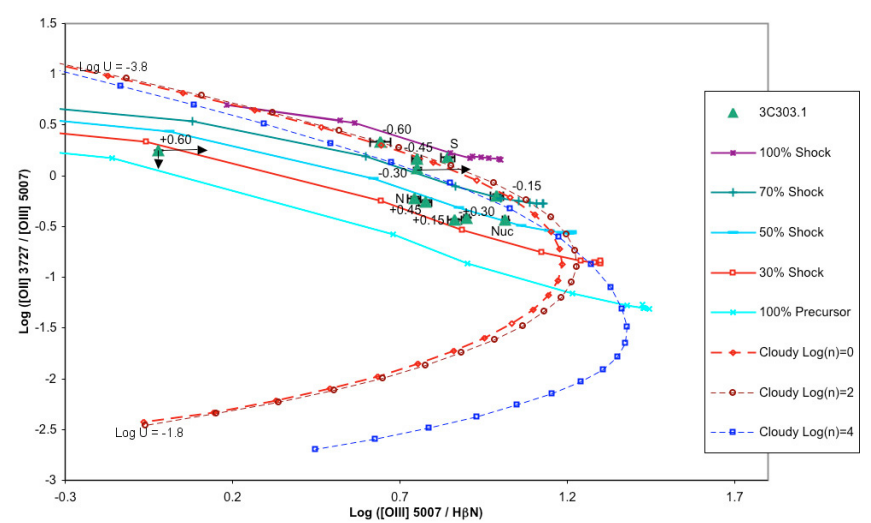

Fig. 6. Same as Fig. 4, but for 3C 303.1.

values of $[\mathrm{O} \mathrm{II}] /[\mathrm{O} \mathrm{III}]$ ) consistent with the nuclei containing the highest ionization gas. The data for the extended emission clearly indicate lower ionization than the nuclei. In addition the data for the extended emission are at higher ionization than the $100 \%$ shock models from MAPPINGS and are at lower ionization than the CLOUDY AGN photo-ionization models. In general the extended emission lines are consistent with a mixture of shocked and photo-ionized gas. In 3C 67, the data lie between the MAPPINGS models for a contribution to the observed luminosity from shocked gas ranging from 0 to $50 \%$. In $3 \mathrm{C} 303.1$, the contribution to the luminosity from shocked gas is between 30 and $70 \%$. In 3C 277.1, the data scatter around the model for $100 \%$ contribution to the luminosity from precursor gas. Thus, the contribution from shocks increases from 3C 277.1 to $3 \mathrm{C} 67$ to $3 \mathrm{C} 303.1$. The sources tend to lie in the regions for moderate to high shock velocities $\left(500\right.$ to $\left.+1000 \mathrm{~km} \mathrm{~s}^{-1}\right)$ and we do not obtain any useful constraints on magnetic field strengths in the MAPPINGS models. We note that these results are consistent with previous work on CSS radio sources using ground based data (Gelderman \& Whittle 1994; Morganti et al. 1997). We also used the observed $\mathrm{H} \alpha / \mathrm{H} \beta$ ratios to test the hypothesis that the low values of the $[\mathrm{O} \mathrm{II}] /[\mathrm{O} \mathrm{III}]$ ratio are produced by large amounts of redenning. However, the observed Balmer ratios (or even values $10 \%$ higher than observed) are not consistent with significant redenning effects on the $[\mathrm{O} \mathrm{II}] /[\mathrm{O} \mathrm{III}]$ ratio (except possibly in the nucleus of 3 C 277.1).

\subsection{Can the central AGN power the emission line luminosity in the extended nebulae?}

We compared the number of ionizing photons produced by the nucleus of the source, with the number of photons needed to produce the observed emission line luminosity (see e.g. Wilson et al. 1988; Baum \& Heckman 1989; Axon et al. 2000; O'Dea et al. 2000). Assuming radiative recombination under case B conditions, the number of ionizing photons $N_{\mathrm{H} \beta}$ needed to produce the observed $\mathrm{H} \beta$ luminosity $L_{\mathrm{H} \beta}$ is:

$N_{\mathrm{H} \beta}=2.1 \times 10^{12} L_{\mathrm{H} \beta}$ photons s$^{-1}$.

We use the integrated [O III] $\lambda 5007$ fluxes measured by Gelderman \& Whittle (1994) (see Table 3) and scale using the typical ratio for the narrow line components in CSS sources 
Table 3. Source properties.

\begin{tabular}{lrrr}
\hline \hline Parameter & 3C 67 & 3C 277.1 & 3C 303.1 \\
\hline ID & $\mathrm{G}$ & $\mathrm{Q}$ & $\mathrm{G}$ \\
redshift & 0.310 & 0.321 & 0.267 \\
scale $(\mathrm{kpc} / \mathrm{arcsec})$ & 4.04 & 4.13 & 3.65 \\
radio power $\log _{10} P_{5 \mathrm{GHz}}\left(\mathrm{Watts} \mathrm{Hz}^{-1}\right)$ & 26.3 & 26.4 & 26.0 \\
angular size $\theta(\mathrm{arcsec})$ & 2.5 & 1.67 & 1.7 \\
linear size $D(\mathrm{kpc})$ & 10.1 & 6.9 & 6.2 \\
integrated emission line flux $F(\mathrm{O}$ III $\lambda 5007)\left(10^{-15} \mathrm{ergs} \mathrm{s}^{-1} \mathrm{~cm}^{-2}\right)$ & 22 & 31 & 28 \\
integrated $\left([\mathrm{O} \mathrm{III} \lambda 5007)\right.$ line width at $50 \%$ of line peak $\left(\mathrm{km} \mathrm{s}^{-1}\right)$ & $600 \pm 42$ & $510 \pm 19$ & $815 \pm 18$ \\
integrated $(\mathrm{O}$ III $\lambda 5007)$ line width at 20\% of line peak $\left(\mathrm{km} \mathrm{s}^{-1}\right)$ & $915 \pm 85$ & $740 \pm 50$ & $1170 \pm 41$ \\
spectral age $(\mathrm{yr})$ & $5 \times 10^{4}$ & $2 \times 10^{5}$ & $1 \times 10^{5}$ \\
advance speed $(\mathrm{v} / \mathrm{c})$ & 0.22 & 0.04 & 0.07 \\
\hline
\end{tabular}

We adopt a Hubble constant of $H_{0}=75 \mathrm{~km} \mathrm{~s}^{-1} \mathrm{Mpc}^{-1}$ and a deceleration parameter of $q_{0}=0.0$. The integrated emission line flux and width are from Gelderman \& Whittle (1994). The spectral age is estimated by fitting a continuous injection model to the integrated radio spectrum and is taken from Murgia et al. (1999). The advance speed is estimated using $2 v=$ linear size/spectral age.

Table 4. Photon counting.

\begin{tabular}{ccccc}
\hline \hline Source & Distance & $\log N_{\mathrm{H} \beta}$ & $\log N_{\mathrm{Nuc}}$ & $N_{\mathrm{H} \beta} / N_{\mathrm{Nuc}}$ \\
\hline 3C 67 & 1620 & 54.4 & 54.3 & 1.3 \\
3C 277.1 & 1700 & 54.6 & 55.1 & 0.35 \\
3C 303.1 & 1350 & 54.4 & 53.1 & 17.0 \\
\hline
\end{tabular}

Source, distance to the source in $\mathrm{Mpc}\left(H_{0}=75 \mathrm{~km} \mathrm{~s}^{-1} \mathrm{Mpc}^{-1}\right)$ and number of photons needed to ionize $\mathrm{H}_{\beta}$, ionizing photons produced by the nucleus, and the ratio between the last two. If the ratio is $\leq 1$, the nucleus is producing enough photons to ionize $\mathrm{H}_{\beta}$, if the ratio is higher than one, another source of ionization, such as shocks, is required.

(see also Gelderman \& Whittle 1994): $\mathrm{H} \beta /[\mathrm{O}$ III $] \lambda 5007=$ $0.18 \pm 0.02$.

The number of photons in the continuum, between frequencies $v_{1}$ and $v_{2}$ are given by:

$N_{\mathrm{Nuc}}=4 \pi D^{2} S_{0}(\alpha h)^{-1}\left(v_{1}^{\alpha}-v_{2}^{\alpha}\right)$ photons s$^{-1}$

where $D$ is the luminosity distance, the flux density spectrum is given by $F_{v}=S_{0} v^{-\alpha}$ (we adopt $\alpha=1$ ) and h is Planck's constant. We are only interested in the photons with enough energy to ionize Hydrogen, so we choose $v_{1}=3.3 \times 10^{15} \mathrm{~Hz}(912 \AA$ or $13.6 \mathrm{eV})$ and $v_{2}=4.8 \times 10^{17} \mathrm{~Hz}(17 \mathrm{keV})$. For our spectral index, $\alpha=1$, higher frequencies do not add a significant number of photons. Note that this analysis is subject to the caveat that the continuum emission may not be emitted isotropically, and the extended nebulae may see a different luminosity than we do (e.g. Penston et al. 1990).

The results are shown in Table 4 . We find that the nucleus apparently produces enough ionizing photons to power the emission line luminosity in 3C 277.1 and possibly 3C 67, but not 3C 303.1. This is consistent with the results from our BPT diagrams.

\subsection{Diagnostic ratios vs. kinematics and distance}

Here we look for additional clues to the nature of the ionization and acceleration of the emission line gas. In shock models, the ionization should be related to the gas kinematics (e.g., Clark et al. 1998), while if the ionization is dominated by AGN photoionization, the ionization level and kinematics should be largely independent.

We have studied the behavior of the diagnostic ratios [O III] 5007/H $\beta$, [O II] $\lambda \lambda 3727+29 /[\mathrm{O}$ III] $\lambda 5007$, [N II] $6583 / \mathrm{H} \alpha,[\mathrm{S} \mathrm{II}] / \mathrm{H} \alpha$ with distance to the nucleus of the source, and the gas kinematics (velocity offset relative to the nucleus, and $F W H M$ ). The kinematic data have been taken from the medium dispersion results (O'Dea et al. 2002) given their superior spectral resolution. The low dispersion data give consistent results. The relationships are plotted in Figs. 7 to 8 and the results are summarized in Table 5. We have applied a Spearman rank test to search for correlations and regard correlations with a significance $\geq 99 \%$ as real.

The three sources show a decrease in ionization with distance from the nucleus (based on [O III] 5007/H $\beta$, [O II $] \lambda \lambda$ 3727+29/[O III]5007). This behavior (high ionization in the nucleus, low in the lobes) can be explained by (1) the influence of the central engine in the inner regions, and a low ionization source, like shocks, further out; and (2) dilution of photons from the nucleus with distance. The sources show a weak trend for ionization to decrease with increasing FWHM and a strong trend for ionization to decrease with increasing velocity offset. This relation between ionization level and the kinematics is consistent with shock ionization and acceleration of the gas (as suggested by O'Dea et al. 2002).

\subsection{Cloud properties}

We have estimated the electron temperature using the [O III $] \lambda 4363 /[\mathrm{O}$ III $] \lambda 5007$ diagnostic lines. Given our limited signal-to-noise we derive an estimate of the $\mathrm{e}^{-}$temperature 

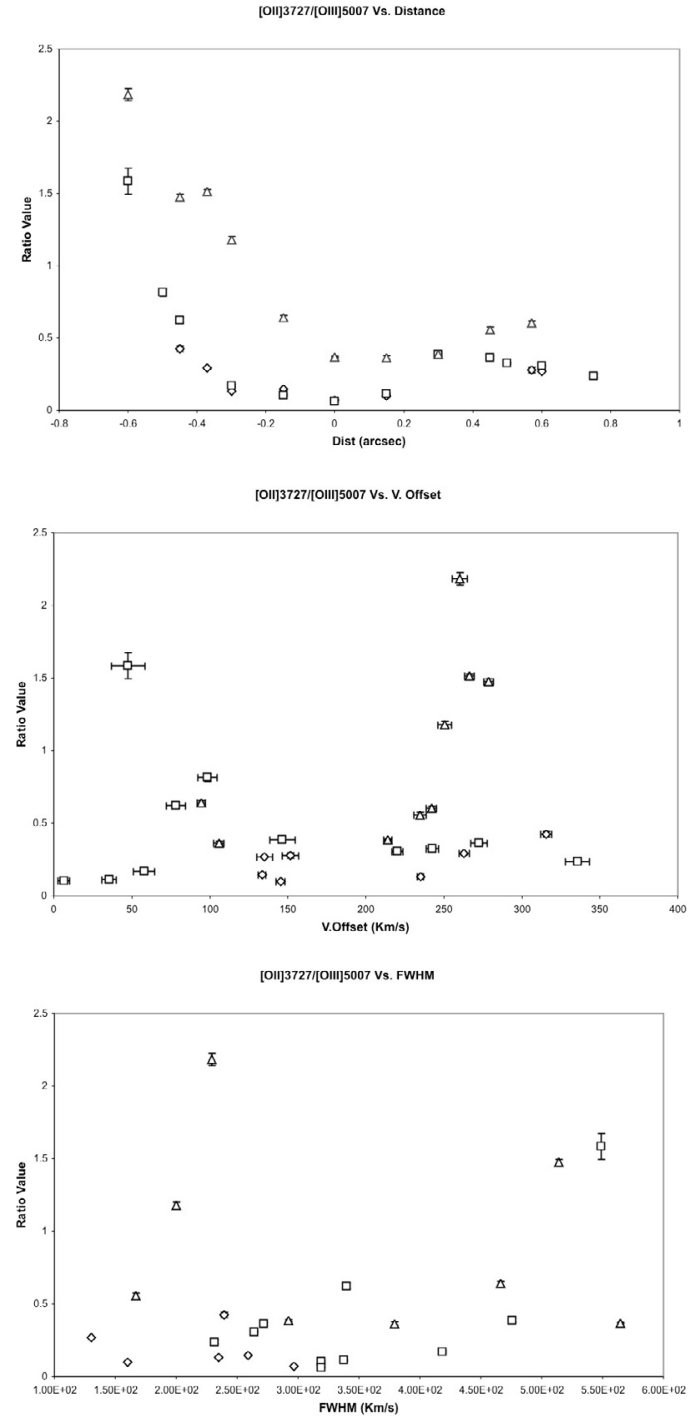

Fig. 7. Behavior of the diagnostic ratio [O II $] \lambda \lambda 3727+29 /[\mathrm{O}$ III] 5007 versus distance, velocity offset and $F W H M$. Diamonds represent 3C 67, squares 3C 277.1 and triangles 3C 303.1.

in the nucleus of 3C 277.1 of $\sim 20000 \mathrm{~K}$ and obtain lower limits $(>7000 \mathrm{~K})$ for the rest of the sources. These temperatures are consistent with previous measurements of temperatures in the nucleus and extended emission-line regions of AGN (Clark et al. 1998; Wilson et al. 1997; Storchi-Bergmann et al. 1996). Tadhunter et al. (1989) showed that the observed ratio O III $\lambda 4363 / \mathrm{O}$ III $\lambda 5007$ implied for several extended emissionline regions electron temperatures ranging from $12800 \mathrm{~K}$ to $22000 \mathrm{~K}$, whereas photoionization models predict much lower values. They discussed several possible explanations for this discrepancy between the results and the models, including additional heating sources such as cosmic rays or shocks, or metal abundances lower than the solar values assumed in the models.

In $3 \mathrm{C} 277.1$ we also found high $\mathrm{H} \alpha / \mathrm{H} \beta$ values for the nucleus $(10.4 \pm 0.2)$ consistent with the measurements of CSS quasars by Baker \& Hunstead (1995), who argued for very dusty gas in these objects. On the other hand, 3C 67 and $3 \mathrm{C} 303.1$ show $\mathrm{H} \alpha / \mathrm{H} \beta$ ratios of $\sim 3.1$.
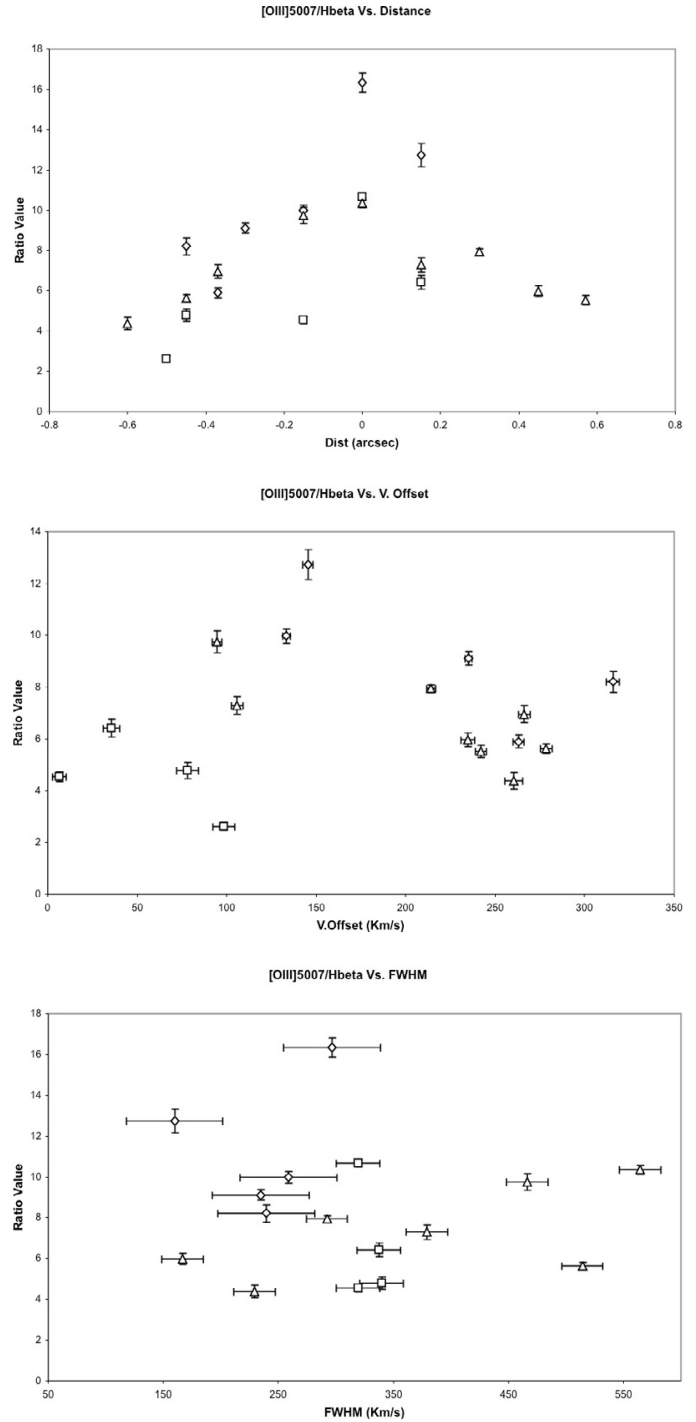

Fig. 8. Behavior of the diagnostic ratio [O III] $5007 / \mathrm{H} \beta$ versus distance, velocity offset and full width half maximum. Diamonds represent 3C 67, squares 3C 277.1 and triangles 3C 303.1.

\section{Summary}

We present Hubble Space Telescope Imaging Spectrograph long-slit spectroscopy of the aligned emission line nebulae in three compact steep spectrum radio sources: 3C 67, 3C 277.1, and $3 \mathrm{C}$ 303.1. In previous papers we have reported evidence that the kinematics of the gas is consistent with being driven by shocks from the expanding radio lobes. Here we present the results of our low dispersion spectra of several diagnostic emission lines. We compare our results with shock and photoionization models and examine the relationships between the ionization diagnostics and the gas kinematics.

We find that the ionization diagnostics are consistent with a mix of shock and photo-ionization in the extended emission line gas. The data for the extended emission are at higher ionization than the $100 \%$ shock models from MAPPINGS and are at lower ionization than the CLOUDY AGN photo-ionization models. In general the extended emission lines are consistent with a mixture of shocked and photo-ionized gas. In 3C 67, 
Table 5. Behavior of diagnostic ratios with distance and kinematics.

\begin{tabular}{|c|c|c|c|c|c|}
\hline Source & Plot & [O III] 5007/H $\beta$ & {$[\mathrm{O}$ II $] /[\mathrm{O} \text { III }]^{a}$} & {$[\mathrm{~N}$ II $] 6583 / \mathrm{H} \alpha$} & {$[\mathrm{S}$ II $] / \mathrm{H} \alpha$} \\
\hline \multirow{3}{*}{$3 \mathrm{C} 67$} & Dist & Decrease $(99 \%)$ & Increase $(95 \%)$ & Asymmetric $^{3}(95 \%)$ & No trend ${ }^{1}$ \\
\hline & VO & Decrease $^{2}(99 \%)$ & Increase $^{2}(85 \%)$ & Increase $^{2}(95 \%)$ & No trend $d^{1,2}$ \\
\hline & $F W H M$ & No Trend & No Trend & No Trend & No trend ${ }^{1}$ \\
\hline \multirow{3}{*}{ 3C 277.1} & Dist & Decrease $^{4}(95 \%)$ & Increase $(99.5 \% \mathrm{~S})$ & Decrease $(90 \% \mathrm{~N})^{5}$ & No trend ${ }^{1}$ \\
\hline & VO & Decrease $^{4}(90 \%)$ & Increase $(90 \% \mathrm{~S})$ & Increase $(95 \% \mathrm{~N})^{5}$ & No trend ${ }^{1}$ \\
\hline & $F W H M$ & No trend ${ }^{4}$ & Increase $(95 \% \mathrm{~S})$ & No trend & No trend ${ }^{1}$ \\
\hline \multirow{3}{*}{ 3C 303.1} & Dist & Decrease $(99.5 \%)$ & Increase $(99 \%)$ & No trend ${ }^{3}$ & Asymmetric $^{3}(99.5 \%)$ \\
\hline & $\mathrm{VO}$ & Decrease $(99 \%)$ & Increase $(99.5 \%)$ & No trend & Increase $(95 \%)$ \\
\hline & $F W H M$ & Increase $(90 \%)$ & No Trend & No trend & No trend \\
\hline
\end{tabular}

${ }^{a}[\mathrm{O}$ II $] \lambda \lambda 3727+29 /[\mathrm{O}$ III $] \lambda 5007$.

1 Very few clear detections.

${ }^{2}$ Most of the points around $|\mathrm{VO}| \sim 150 \mathrm{~km} \mathrm{~s}^{-1}$.

${ }^{3}$ Increases to the south. Decreases to the north.

${ }^{4}$ Few clear detections of $\mathrm{H} \beta$.

${ }^{5}$ Few clear detections of $[\mathrm{N}$ II] in the South Lobe.

Summary of the behavior of the different ratios with distance to the nucleus, velocity offset (relative to the nucleus) and FWHM. First column gives the name of the source, second column the " $X$ " axis in the plot; the next four columns give the studied ratios. Asymmetric: significantly different values in the two lobes. No trend: no observable trend. Increase/Decrease: trend of the ratio with increasing values of distance, velocity offset or FWHM. Where trends were found, we note the - minimum - confidence level based on the Spearman's Rank Correlation for that plot. A letter $\mathrm{S}$ or $\mathrm{N}$ means the correlation was only found in the southern $(\mathrm{S})$ or northern $(\mathrm{N})$ lobe. See the text for more detailed comments on each ratio and source.

the data lie between the MAPPINGS models for a contribution to the observed luminosity from shocked gas ranging from 0 to $50 \%$. In 3C 303.1, the contribution to the luminosity from shocked gas is between 30 and $70 \%$. In 3C 277.1, the data scatter around the model for $100 \%$ contribution to the luminosity from precursor gas. The sources tend to lie in the regions for moderate to high shock velocities (500 to $1000 \mathrm{~km} \mathrm{~s}^{-1}$ ) and we do not obtain any useful constraints on magnetic field strengths in the MAPPINGS models.

The three sources show a decrease in ionization with distance from the nucleus (consistent with a decrease in photoionization with distance) a weak trend for ionization to decrease with increasing FWHM and a strong trend for ionization to decrease with increasing velocity offset (which is consistent with shock ionization).

These results are consistent with a picture in which the CSS sources interact with dense clouds as they propagate through their host galaxies, shocking the clouds thereby ionizing and accelerating them (as suggested by O'Dea et al. 2002).

Acknowledgements. Support for this work was provided by NASA through grant number GO-08104.01-97A (PI C. O'Dea) from the Space Telescope Science Institute, which is operated by the Association of Universities for Research in Astronomy, Inc., under NASA contract NAS5-26555. These observations are associated with program 8104. WDV's work was performed under the auspices of the US Department of Energy, National Nuclear Security Administration by the University of California, Lawrence Livermore National Laboratory under contract No. W-7405-Eng-48. This research has made use of the NASA/IPAC Extragalactic Database (NED) which is operated by the Jet Propulsion Laboratory, California
Institute of Technology, under contract with the National Aeronautics and Space Administration. This research has made use of NASA's Astrophysics Data System. We thank the referee, Dr. Ignas Snellen, for useful comments on the manuscript.

\section{References}

Alexander, P., \& Leahy, J. P. 1987, MNRAS, 225, 1

Axon, D. J., Capetti, A., Fanti, R., et al. 2000, AJ, 120, 2284

Baker, J. C., \& Hunstead, R. W. 1995, ApJ, 452, L95

Baldwin, J. A., Phillips, M. M., \& Terlevich, R. 1981, PASP, 93, 5

Baum, S. A., \& Heckman, T. 1989, ApJ, 336, 702

Begelman, M. C. 1996, in Cygnus A - Study of a Radio Galaxy, 209

Bicknell, G. V., Dopita, M. A., \& O’Dea, C. P. O. 1997, ApJ, 485, 112

Cardelli, J. A., Clayton, G. C., \& Mathis, J. S. 1989, ApJ, 345, 245

Carvalho, J. C. 1994, A\&A, 292, 392

Carvalho, J. C. 1998, A\&A, 329, 845

Clark, N. E., Axon, D. J., Tadhunter, C. N., Robinson, A., \& O’Brien, P. 1998, ApJ, 494, 546

de Vries, W. H., O’Dea, C. P., Baum, S. A., \& Barthel, P. D. 1999, ApJ, 526, 27

de Vries, W. H., O’Dea, C. P., Baum, S. A., et al. 1997, ApJS, 110, 191

De Young, D. S. 1993, ApJ, 402, 95

De Young, D. S. 1997, ApJ, 490, L55

Dopita, M. A., \& Sutherland, R. S. 1996, ApJS, 102, 161

Eracleous, M., \& Halpern, J. P. 2004, ApJS, 150, 181

Fanti, C., Fanti, R., Dallacasa, D., et al. 1995, A\&A, 302, 317

Fanti, C., Pozzi, F., Dallacasa, D., et al. 2001, A\&A, 369, 380

Fanti, R., Fanti, C., Schilizzi, R. T., et al. 1990, A\&A, 231, 333

Ferland, G. J., Korista, K. T., Verner, D. A., et al. 1998, PASP, 110, 761

Gelderman, R., \& Whittle, M. 1994, ApJS, 91, 491 
Kaiser, C. R., \& Alexander, P. 1997, MNRAS, 286, 215

Kaiser, C. R., Dennett-Thorpe, J., \& Alexander, P. 1997, MNRAS, 292, 723

Kewley, Dopita, Evans, et al. 2003, in preparation

Kriss, G. 1994, in Astronomical Data Analysis Software and Systems III, ASP Conf. Ser., 61, 437

Morganti, R., Tadhunter, C. N., Dickson, R., \& Shaw, M. 1997, A\&A, 326,130

Moy, E., \& Rocca-Volmerange, B. 2002, A\&A, 383, 46

Murgia, M., Fanti, C., Fanti, R., et al. 1999, A\&A, 345, 769

O'Dea, C. P. 1998, PASP, 110, 493

O'Dea, C. P., \& Baum, S. A. 1997, AJ, 113, 148

O'Dea, C. P., Baum, S. A., \& Stanghellini, C. 1991, ApJ, 380, 66

O'Dea, C. P., de Vries, W. H., Koekemoer, A. M., et al. 2003, Publ. Astron. Soc. Austr., 20, 88

O'Dea, C. P., de Vries, W. H., Koekemoer, A. M., et al. 2002, AJ, 123, 2333

O’Dea, C. P., De Vries, W. H., Worrall, D. M., Baum, S. A., \& Koekemoer, A. 2000, AJ, 119, 478
Osterbrock, D. E. 1989, Astrophysics of gaseous nebulae and active galactic nuclei, Research supported by the University of California, John Simon Guggenheim Memorial Foundation, University of Minnesota, et al. (Mill Valley, CA: University Science Books), 422 Penston, M. V., Robinson, A., Alloin, D., et al. 1990, A\&A, 236, 53 Readhead, A. C. S., Taylor, G. B., Pearson, T. J., \& Wilkinson, P. N. 1996a, ApJ, 460, 634

Readhead, A. C. S., Taylor, G. B., Xu, W., et al. 1996b, ApJ, 460, 612 Schlegel, D. J., Finkbeiner, D. P., \& Davis, M. 1998, ApJ, 500, 525 Snellen, I. A. G., Schilizzi, R. T., Miley, G. K., et al. 2000, MNRAS, 319,445

Storchi-Bergmann, T., Wilson, A. S., Mulchaey, J. S., \& Binette, L. 1996, A\&A, 312, 357

Tadhunter, C. N., Robinson, A., \& Morganti, R. 1989, in Extranuclear Activity in Galaxies, 293

Veilleux, S., \& Osterbrock, D. E. 1987, ApJS, 63, 295

Wilson, A. S., Binette, L., \& Storchi-Bergmann, T. 1997, ApJ, 482, L131

Wilson, A. S., Ward, M. J., \& Haniff, C. A. 1988, ApJ, 334, 121 